\title{
Gene Editing For Duchenne Muscular Dystrophy: Promise and Challenges for Clinical Application
}

\section{Mohammadsharif Tabebordbar*}

Editas Medicine, Cambridge, MA, USA

Keywords: Gene editing; Clinical application; Diseases; mRNA; Stem cells; Virus

\section{Editorial}

Recent advances in the gene editing technology has sparked enthusiasm and promise for developing novel therapeutics for a wide range of genetic diseases. Different research groups have provided proof of concept evidence for the use of clustered regularly interspaced short palindromic repeats (CRISPR)-Cas9 gene editing system to modify disease-associated alleles both in cell culture and in animal models.

Monogenic diseases with defined underlying genetic cause, such as sickle cell anemia, cystic fibrosis and Duchenne Muscular Dystrophy (DMD) are among the primary targets for gene editingbased therapeutics. DMD is caused by frame-disrupting mutations in the DMD gene encoding for dystrophin protein. Gene editing-based approaches have been used to modify the mutated DMD gene, aimed at restoring the mRNA reading frame and expression of a truncated but functional dystrophin protein, in cultured myoblasts and induced pluripotent stem cells (iPSCs) from DMD patients [1-3] and also in muscles and muscle stem cells of the mdx mouse model [4-6].

Large size of the dystrophin coding sequence $(\sim 11 \mathrm{~kb})$ is an obstacle for packaging the full length gene in adeno associated virus (AAV) vectors and only miniaturized versions of the gene (microdystrophin) can be delivered in vivo using AAVs. Gene editing has the potential to address several limitations of conventional microdystrophin gene augmentation therapy as the dystrophin protein restored by this approach is closer to the full length protein in size. Dystrophin expression in gene edited cells is regulated by endogenous regulatory elements present in the DMD locus, which allows for expression of different mRNA isoforms and is not subject to transgene silencing, as opposed to microdystrophin transgene expression from the viral genome; And permanent modification of the mutated DMD gene in muscle stem cells and their myoblast progenies by gene editing can provide a reservoir of progenitor cells capable of producing dystrophinexpressing muscle fibers, while episomal AAV genome encoding for microdystrophin is diluted from proliferating muscle stem cells and myoblasts during muscle regeneration.

Nevertheless, important challenges remain to be addressed for therapeutic application of gene editing for DMD. CRSIPR/Cas9 can generate unwanted mutations at off-target genomic sites different from the on-target sequence $[7,8]$. These off-target mutations can be the source of potential complications for therapeutic applications of the technology. Recent advances in the field of genome engineering have resulted in development of different strategies to reduce genome-wide off-target mutations of Cas9 endonuclease. These strategies include reducing the length of the guide sequence in guide RNAs (gRNAs) [9], using paired Cas9 nickases [10], and developing Cas9 variants with amino acid substitutions in the DNA binding domains that show lower off-target rates [11,12]. However, there is still need for improving the specificity of Cas 9 and investigating the potential accumulation of off- target mutations due to continuous Cas9 expression in post mitotic cells before making further steps towards clinical application of the technology.

Expression of the bacterial Cas9 protein in mouse muscle has been reported to induce cellular and humoral immune response in the animals [13], which highlights the requirement for more careful analysis of the potential immune response complications as a result of Cas9 expression in mammalian tissues. The use of immunosuppressants and non-viral delivery methods that provide the possibility for transient expression of Cas 9 can be used to avoid long-term expression of the protein and induction of a chronic immune response. Furthermore, identifying immunodominant epitopes of the protein can help to engineer Cas9 variants with lower immunogenicity.

Levels of gene targeting in muscle stem cells are currently rather low, suggesting a need to investigate novel AAV serotypes to identify those with optimal tropism for muscle satellite cells. Directed evolution and in vivo selection has been recently used to engineer novel AAV capsids with high tropism for tissues that are difficult to transduce with naturally occurring AAVs, such as the outer retina [14] and human hepatocytes [15]. Therefore, using a directed evolution and in vivo selection strategy for generating novel AAV serotypes with high tropism for satellite cells can be considered as an exciting next step. Another strategy to increase the efficiency of gene editing in muscle fibers and muscle stem cells is to optimize single AAV vectors encoding for gene editing components by identifying compact and strong muscle specific promoters and highly efficient and specific guide RNAs.

Furthermore, efficacy of DMD gene editing reagents, off-target mutations and potential immune response to Cas9 need to be evaluated in large DMD animal models (e.g. canine models of the disease) as well.

In conclusion, gene editing holds great promise as an effective therapeutic approach for treating DMD, however, overcoming the above-mentioned challenges would be essential for developing efficient and safe CRISPR medicines for the disease.

\section{References}

1. Ousterout DG, Kabadi AM, Thakore PI, Majoros WH, Reddy TE, et al. (2015) Multiplex CRISPR/Cas9-based genome editing for correction of dystrophin

*Corresponding author: Mohammadsharif Tabebordbar, Editas Medicine, Cambridge, MA, USA, Tel: 617.401.9054; E-mail: sh.bordbar@gmail.com

Received December 15, 2016; Accepted December 18, 2016; Published May 31, 2018

Citation: Tabebordbar M (2018) Gene Editing For Duchenne Muscular Dystrophy Promise and Challenges for Clinical Application. Stem Cell Res Regen Med 1: e102.

Copyright: @ 2018 Tabebordbar M. This is an open-access article distributed unde the terms of the Creative Commons Attribution License, which permits unrestricted use, distribution, and reproduction in any medium, provided the original author and source are credited. 
Citation: Tabebordbar M (2018) Gene Editing For Duchenne Muscular Dystrophy: Promise and Challenges for Clinical Application. J Stem Cell Res Ther: e121.

mutations that cause Duchenne muscular dystrophy. Nat Commun 6: 6244 . [PubMed]

2. Hongmei LL, Naoko F, Noriko S, Saya S, Tokiko O, et al. (2015) Precise correction of the dystrophin gene in duchenne muscular dystrophy patient induced pluripotent stem cells by TALEN and CRISPR-Cas9. Stem Cell Reports 4: 143-154. [PubMed]

3. Courtney SY, Michael RH, Natalia VE, Haruko N, Majib J, et al. (2016) A single CRISPR-Cas9 deletion strategy that targets the majority of DMD patients restores dystrophin function in hiPSC-derived muscle cells. Cell Stem Cell 18: 533-540. [PubMed]

4. Tabebordbar M, Zhu K, Cheng JK, Chew WL, Widrick JJ, et al. (2016) In vivo gene editing in dystrophic mouse muscle and muscle stem cells. Science 351 407-411. [PubMed]

5. Christopher EN, Chady HH, David GO, Pratiksha IT, Eirik AM, et al. (2006) In vivo genome editing improves muscle function in a mouse model of Duchenne muscular dystrophy. Science 351: 403-407. [PubMed]

6. Long C, Amoasii L, Mireault AA, McAnally JR, Li H, et al. (2016) Postnata genome editing partially restores dystrophin expression in a mouse model of muscular dystrophy. Science 351: 400-403. [PubMed]

7. Fu Y, Foden JA, Khayter C, Maeder ML, Reyon D, et al. (2013) High-frequency off-target mutagenesis induced by CRISPR-Cas nucleases in human cells. Nat Biotechnol 31: 822-826. [PubMed]

8. Hsu PD, Scott DA, Weinstein JA, Ran FA, Konermann S, et al. (2013) DNA targeting specificity of RNA-guided Cas9 nucleases. Nat Biotechnol 31: 827 832. [PubMed]

9. Fu Y, Sander JD, Reyon D, Cascio VM, Joung JK (2014) Improving CRISPRCas nuclease specificity using truncated guide RNAs. Nat Biotechnol 32: 279284. [PubMed]

10. Ran FA, Hsu PD, Lin CY, Gootenberg JS, Konermann S, et al. (2013) Double nicking by RNA-guided CRISPR Cas9 for enhanced genome editing specificity Cell 154: 1380-1389. [PubMed]

11. Ian MS, Linyi G, Bernd Z, David AS, Winston XY, et al. (2016) Rationally engineered Cas9 nucleases with improved specificity. Science 351: 84-88. [PubMed]

12. Kleinstiver BP, Pattanayak V, Prew MS, Tsai SQ, Nguyen NT, et al. (2016) Highfidelity CRISPR-Cas9 nucleases with no detectable genome-wide off-target effects. Nature 529: 490-495. [PubMed]

13. Chew WL, Tabebordbar M, Cheng JK, Mali P, Wu EY, et al. (2016) A multifunctional AAV-CRISPR-Cas9 and its host response. Nat Methods 13 868-874. [PubMed]

14. Dalkara D, Byrne LC, Klimczak RR, Visel M, Yin L, et al. (2013) In-vivo directed evolution of a new adeno-associated virus for therapeutic outer retinal gene delivery from the vitreous. Sci Transl Med 5: 189ra76. [PubMed]

15. Leszek L, Allison PD, Kirk C, Yue Z, Sharon CC, et al. (2014) Selection and evaluation of clinically relevant AAV variants in a xenograft liver model. Nature 506: 382-386. 